\section{In vivo confocal microscopy of toxic keratopathy}

\begin{abstract}
Purpose To explore the morphological characteristics of toxic keratopathy (TK), which clinically presented as superficial punctate keratopathy (SPK), with the application of in vivo laser-scanning confocal microscopy (LSCM), and evaluate its potential in the early diagnosis of TK.

Patients and methods This was a crosssectional study involving 16 patients with TK and 16 patients with dry eye (DE), demonstrating SPK under slit-lamp observation, and 10 normal eyes were enrolled in the study. All participants underwent history interviews, fluorescein staining, tear film break-up time (BUT) tests, Schirmer tests, and in vivo LSCM.

Results The area grading of corneal fluorescein punctate staining was higher in the TK group than the DE group. Measured by in vivo LSCM, superficial epithelial cell density was lower in the TK group than that of DE group. The sub-basal nerve presented lower density and tortuosity in the TK group than the DE group. Most notably, deposits with a snow-like appearance were observed in the epithelium in $12 / 16(75.0 \%)$ of the TK cases, but none in the DE group $(P<0.05)$. Conclusion The SPK in TK patients was characterized by more widespread punctate staining, a lower density of superficial epithelial cells and sub-basal nerves, and a typical snow-like pattern of deposits in the epithelium by LSCM. These features might facilitate early diagnosis of TK from other disorders manifested as SPK.

Eye (2017) 31, 140-147; doi:10.1038/eye.2016.213; published online 14 October 2016
\end{abstract}

Department of

Ophthalmology, Eye, Ear, Nose, and Throat Hospital, School of Shanghai Medicine, Fudan University, Shanghai, China

Correspondence: J Xu, Department of Ophthalmology, Eye, Ear, Nose and Throat Hospital, School of Shanghai Medicine, Fudan University, 83 Fenyang Road, Shanghai 200031, China Tel: +86 013816881607; Fax: +8602164377151. Email: jianjiangxu@126.com

Received: 27 March 2016 Accepted in revised form: 31 August 2016 Published online: 14 October 2016

\section{Introduction}

Toxic keratopathy (TK) involves corneal damage or dysfunction that results from exposure to substances that are disruptive to corneal anatomy or metabolism. The most common cause of TK is the use of ophthalmic medications, although a wide variety of chemicals and systemic medications can also cause TK. ${ }^{1}$ Cases of drug-induced TK have been prevalent in ophthalmic clinics for two reasons. On the one hand, most of the topically applied drugs, either prescribed or sold over-the-counter, are capable of causing corneal damage at sufficient concentrations. ${ }^{2}$ Patients who have glaucoma, viral keratitis, keratoconjunctivitis sicca or other ocular surface conditions, generally need multidrug remedies, and these pre-existing conditions may especially predispose them to drug toxicity. The preservative in the eye drops, mainly benzalkonium chloride (BAC), is another important cause for epithelial lesions. On the other hand, the use of eye drops for a week or more may cause TK, which can often be confused with the worsening of the patient's initial disease and result in incorrect aggressive management, which can lead to a medical cascade effect, even causing persistent epithelial defects that can result in poor vision. ${ }^{3}$ Therefore, the early recognition of TK is critical for a better prognosis.

The symptoms of TK, including hyperemia, chemosis, photophobia, and eye pain, are usually non-specific and can also be found in patients with other disorders such as dry eye (DE). ${ }^{4}$ It generally presents as superficial punctate keratopathy (SPK) at early stage of clinical course, ${ }^{2}$ which is featured by the presence of elevated epithelial lesions with round- or oval-shaped clusters of gray granular opacities ${ }^{5}$ and should be distinguished from Thygeson superficial punctate keratitis (TSPK). In general, the 'spots' represent the loss or detachment of epithelial cells as a result of various types of damage to the corneal epithelium. Apart from TK, SPK can also be detected in patients with DE, meibomian gland dysfunction, neurotrophic keratopathy or exposure keratopathy, and contact lenses wearers. ${ }^{6}$ Therefore, the identification of the etiology of SPK is vital to differentiate TK from other disorders. 
There were very few studies that investigated the morphological features of SPK in patients with TK. In the current study, with the application of in vivo laserscanning confocal microscopy (LSCM), we attempted to identify the characteristics of SPK in patients diagnosed as either TK or DE, and explore the potential of LSCM in the early diagnosis of TK.

\section{Methods}

\section{Subjects}

Thirty-two subjects with corneal fluorescein punctate spots who visited the Department of Ophthalmology at EENT Hospital of Fudan University from 13 October 2012 to 1 April 2014 were enrolled in this study. Ten healthy subjects were also included as controls. This study was approved by the ethics committee of the hospital and was conducted according to the tenets of the Declaration of Helsinki. Written informed consent was obtained from all subjects. Each participant was interviewed about the medical history before ocular examinations. One eye was randomly selected if the patient was binocularly affected. On the basis of their medical history and clinical examinations, each patient was either diagnosed with DE or TK. Patients who had punctate fluorescein staining were classified as suspected TK if they had a history of two or more types of eye drops use for at least one month, and were diagnosed as TK if they showed improvements in both symptoms and clinical signs 2-4 weeks after stopping the use of all eye drops, except for preservativefree artificial tears (Figure 1). The diagnosis of DE was made according to the Japanese DE diagnostic criteria $(2005)^{7,8}$ because the specificity of diagnosis rather than the sensitivity was more concerned in this study, In brief, DE was diagnosed on the following criteria: (1) a Schirmer's test result of $<5 \mathrm{~mm}$, or a BUT test result of $<5 \mathrm{~s}$; (2) conjunctival and corneal epithelial fluorescein staining $>1$ point (excluding all other etiologies); and
(3) the presence of DE symptoms. Moreover, all patients diagnosed with $\mathrm{DE}$ met the requirement that their condition improve with continuing anti-inflammatory drug treatment. Subjects with a history of ocular trauma, thermal/chemical injuries, wearing contact lenses during the past 6 months, occupational exposure to intense ultraviolet rays, conjunctivitis, infectious keratitis, trichiasis/distichiasis, entropion/ectropion, hypophasis, flabby upper eyelid or meibomian gland dysfunction at the time of their clinical examination were excluded.

\section{Clinical assessment}

The ocular symptom and medication history of each patient was recorded. After that, each patient completed the Ocular Surface Disease Index (OSDI) questionnaire and received a slit-lamp examination. Tear film break-up time (BUT) tests and fluorescein staining were performed during the slit-lamp examinations. Schirmer's I tests were performed without anesthesia after the slit-lamp examinations to avoid any effects on the corneal epithelium. The grade of corneal fluorescein punctate staining was classified according to the area and density (AD) classification system devised by Miyata et al. ${ }^{6}$ After fluorescein staining, the total sum of the area of SPK was graded from A0 through A3, and the density was graded from D0 through D3. The grade of severity was calculated as the area grade multiplied by the density grade. With regard to the distribution of the staining, the entire cornea was divided into the following three parts: the upper $1 / 3$, the middle $1 / 3$, and the lower $1 / 3$. The involvement rates of the three parts were respectively calculated and compared.

\section{In vivo LSCM}

After a detailed explanation of the procedure, the subject underwent in vivo LSCM examination using a Heidelberg retina tomograph (HRTII)/Rostock cornea module (RCM)
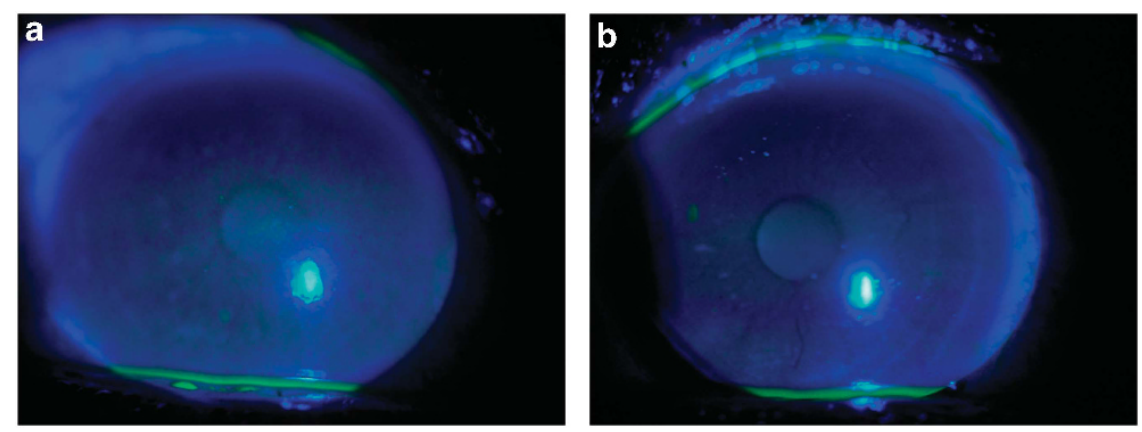

Figure 1 Corneal Fluorescein Staining of a TK Patient. Representative corneal fluorescein staining pictures were taken in TK patients on Day 0 (a), and 2 weeks after patients stopped all previous eye drops application. (b) The images show a decrease in both the area and the density of fluorescein staining. 
(Heidelberg Engineering GmbH, Dossenheim, Germany), that had a 60water-immersion objective lens (Olympus Europa GmbH, Hamburg, Germany) and a 670-nm diode laser as a light source. The lateral and longitudinal optical resolutions of the system were both $1 \mu \mathrm{m}$, and the amplitude of the field was $384 \times 384 \mu \mathrm{m}\left(147456 \mu \mathrm{m}^{2}\right)$. The cornea was anesthetized with one drop of oxybuprocaine hydrochloride $0.4 \%$ (Benoxil; Santen Pharmaceutical, Osaka, Japan). The scan sites were generally in the central cornea or the lower $1 / 3$ part of the cornea, where the fluorescein staining was the most intensive, and the images were recorded at one point along the $z$-axis using the single-scan mode (with a $1 \mu \mathrm{m}$ step size in depth). All the images were evaluated by two independent investigators (QL, YC) in a masked manner to ensure objective analysis. The best-focused and most representative images were selected. Three images concerning a specific corneal layer were selected for analysis, and the densities were averaged for each patient. The superficial and basal layer epithelial cells, sub-basal nerve plexus, Langerhans cells, and stromal keratocytes were quantitatively analyzed. Image J software (NIH, Bethesda, MD, USA) was used to calculate the cell density (number of cells/area) and the nerve density(sum of nerve length/area). The grade of nerve tortuosity was classified in four grades according to a scale used by Oliveira-Soto et $a l^{9}$ Qualitative abnormalities were also described and compared.

\section{Statistical analysis}

SPSS 16.0 (SPSS Inc., Chicago, IL, USA) was used for statistical analysis. The normal distribution of the variables was determined with the Kolmogorov-Smirnov test. The quantitative variables were expressed as the mean and SD, and the qualitative variables were expressed as a frequency. The inter-rater reliability was examined by intra-class correlation coefficient. The ANOVO test, followed by Bonferroni if the data has homogeneity of variance and Dunnett T3 If the variance is not homogeneous, was performed to evaluate the continuous quantitative variables, and the MannWhitney $U$-test was used to evaluate the categorical variables. The Fisher's exact text was used to analyze the qualitative variables. Test results are two-tailed, where $P<0.05$ is considered statistically significant.

\section{Results}

\section{Demographics}

A total of 32 patients with corneal fluorescein punctate staining were enrolled in this study, including 16 patients diagnosed as TK and 16 patients diagnosed as DE. Ten eyes of normal subjects were also evaluated. The mean ages of patients with TK, patients with DE, and normal subjects were $52.4 \pm 19.9(16-76)$ years, $42.9 \pm 12.9$ (17-67) years, and $42.7 \pm 12.3(23-57)$ years $(P>0.05)$, respectively. The majority of patients were female in all groups $(56.3 \%$ in the TK group, $87.5 \%$ in the DE group and $60 \%$ in the normal; $P>0.05)$. Since previous study has shown that there was no significant difference in the cell densities of any corneal layer between female and male patients, ${ }^{10}$ we assumed that the gender imbalance among the three groups did not influence the results of our comparisons.

\section{Clinical characteristics}

The area grade (0-3) of the corneal fluorescein punctate staining was higher in the TK group than in the DE group $(2.63 \pm 0.50$ vs $1.87 \pm 0.62, P<0.05)$. This finding accounted for the difference observed in the severity grade (0-9) between the two groups $(7.06 \pm 1.88$ vs $4.00 \pm 1.93$, $P<0.05)$ because there was no significant difference in the density grade between the two groups (Table 1).With regard to the distribution of the staining, the whole cornea was divided into the following three parts: the upper $1 / 3$, the middle $1 / 3$, and the lower $1 / 3$. The involvement rates of the three parts in the TK group were 43.8, 93.8, and 93.8\% respectively. Although it was 18.8, 68.8, and 93.8\%, respectively, in the DE group. This result implies that corneal epithelial defect in both groups was mainly located in the middle and lower part of the cornea (Table 2).

\section{LSCM characteristics}

The inter-rater reliability between (QL) and (YC) was very high with a $91 \%$ agreement rate. Therefore, the average of the two investigators' data was considered for analysis. The cell density of the superficial epithelium was lower in the TK group $\left(845.84 \pm 193.89 / \mathrm{mm}^{2}\right)$ than that of the DE group $\left(1055.67 \pm 176.47 / \mathrm{mm}^{2}, P<0.05\right)$ and the normal group (1426.34 $\pm 188.50, P<0.05)$ (Figure $2 a)$. However, there was no significant difference of the basal epithelial cell density between the TK group $(5353.46 \pm 1039.21$ / $\left.\mathrm{mm}^{2}\right)$ and the DE group $\left(5384.37 \pm 1098.56 / \mathrm{mm}^{2}\right)$,

Table 1 Grading of corneal fluorescein punctate staining

\begin{tabular}{lccc}
\hline Parameter & $\begin{array}{c}\text { TK } \\
\text { Mean } \pm S D\end{array}$ & $\begin{array}{c}\text { DE } \\
\text { Mean } \pm S D\end{array}$ & P-value $^{\text {a }}$ \\
\hline Area & $2.63 \pm 0.50$ & $1.87 \pm 0.62$ & $0.003^{*}$ \\
Density & $2.69 \pm 0.49$ & $2.19 \pm 0.75$ & 0.073 \\
Severity & $7.06 \pm 1.88$ & $4.00 \pm 1.93$ & $0.000^{*}$ \\
\hline
\end{tabular}

${ }^{*} P<0.05$. ${ }^{a} P$-values derived from Mann-Whitney $U$-test. 
or between the TK group and the normal group $\left(5401.18 \pm 622.50 / \mathrm{mm}^{2}\right.$, Figure $\left.2 b\right)$. The sub-basal nerve density of the TK group $\left(1.64 \pm 1.96 \mathrm{~mm} / \mathrm{mm}^{2}\right)$ was lower than that of the DE group $\left(11.88 \pm 3.16 \mathrm{~mm} / \mathrm{mm}^{2}\right.$, $P<0.05)$ and the normal group $\left(9.46 \pm 1.55 \mathrm{~mm} / \mathrm{mm}^{2}\right.$, $P<0.05)$. The tortuosity of the sub-basal nerves was $0.46 \pm 0.44$ in the TK group, which was lower than that of the DE group $(2.55 \pm 0.69, P<0.05)$ and the normal group $(1.95 \pm 0.47, P<0.05)$. These findings demonstrate that there were significant nerve changes in the TK

Table 2 Distribution of corneal fluorescein punctate staining

\begin{tabular}{lccr}
\hline Area & $T K$ & $D E$ & P-value $^{\text {a }}$ \\
\hline Upper & $7 / 16(43.8 \%)$ & $3 / 16(18.8 \%)$ & 0.252 \\
Middle & $15 / 16(93.8 \%)$ & $11 / 16(68.8 \%)$ & 0.172 \\
Lower & $15 / 16(93.8 \%)$ & $15 / 16(93.8 \%)$ & $>0.999$ \\
\hline
\end{tabular}

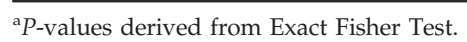

(Figures 2c and d). Interestingly, the sub-basal nerve density of the DE group seemed higher than that of the normal, although there was no significant difference $(P>0.05)$. However, the tortuosity was significantly higher in the DE group than in the normal $(1.95 \pm 0.47$, $P<0.05)$. In addition, there was no significant difference found between the TK group and other groups in the keratocyte density or the Langerhans cell density (Figures 2e and f).

In accordance with lower density of the corneal superficial epithelial cells observed in the TK group, it showed a larger superficial epithelial cell size (Figure 2g) than that of the DE (Figure 2h) and the normal (Figure 2i) groups. There was no difference in the size or density of the basal epithelial cells between the TK group (Figure $2 \mathrm{j}$ ) and the DE group (Figure 2k), or between the TK group and the normal group (Figure 21). The sub-basal nerves of the TK group (Figure $2 \mathrm{~m}$ ) were less intensive and less a

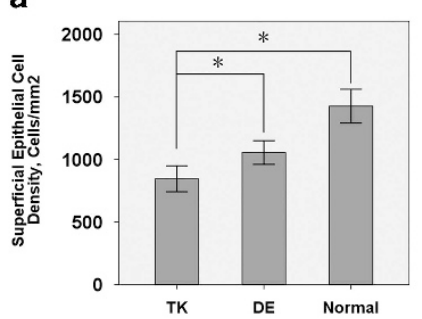

c

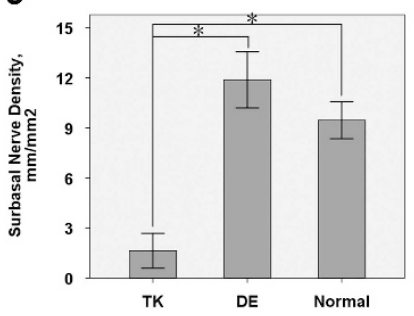

e

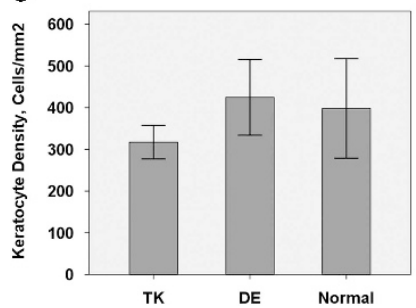

b

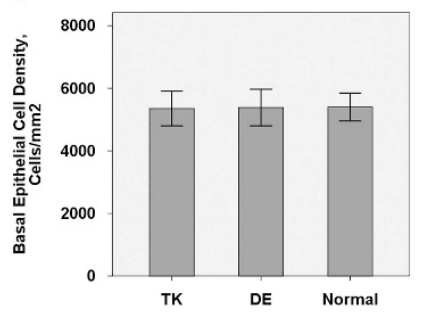

d

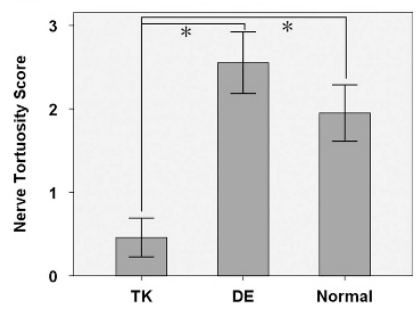

f

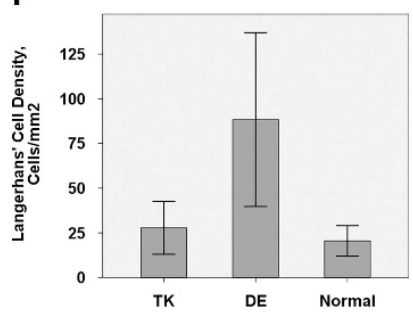

9

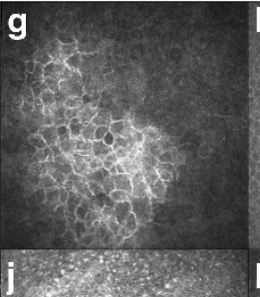

h
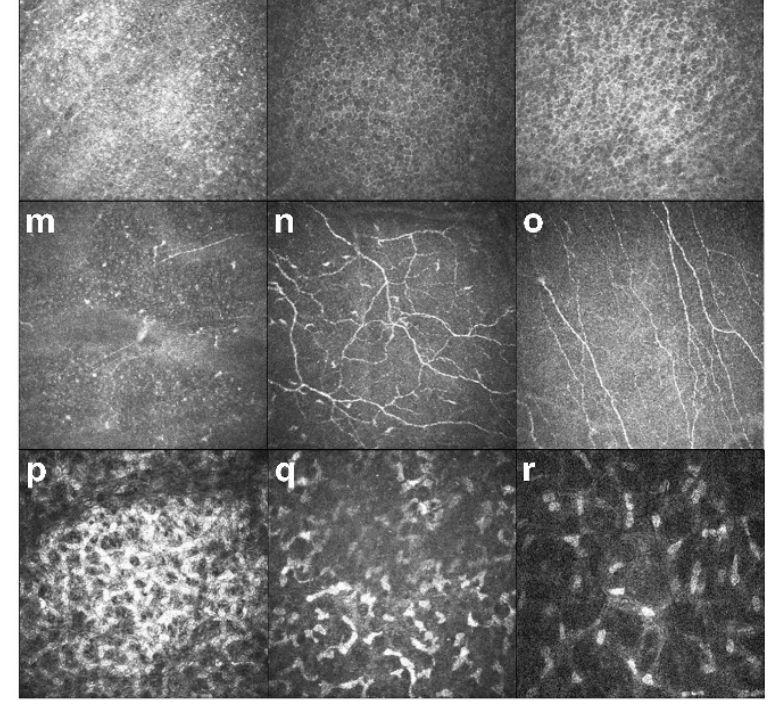

Figure 2 In vivo LSCM Measurements of TK, DE, and the Normal. The cell density of the superficial epithelium was lower for the TK group than for the DE group and for the normal group (a). However, the basal epithelial cell density was not significantly different between the TK group and the DE group, or between the TK group and the normal group (b).The sub-basal nerve density and tortuosity of the TK group was lower than that of the DE group and of the normal group (c, d). In addition, the keratocyte density and the Langerhans cell density of the TK group had no significant difference compared with other groups e, f). *indicates $P<0.05$. Representative in vivo LSCM images of the superficial epithelium ( $\mathrm{g}, \mathrm{h}, \mathrm{i})$, basal epithelium $(\mathrm{j}, \mathrm{k}, \mathrm{l})$, sub-basal nerves (m, n, o), and stromal keratocytes $(p, q, r)$ collected from patients in the TK group $(g, j, m, p)$, patients in the DE group $(h, k, n, q)$, and the controls $(\mathrm{i}, \mathrm{l}, \mathrm{o}, \mathrm{r})$ were shown. 
tortuous than those of the DE group (Figure 2n) and those of the normal group (Figure 2o). Keratocytes were largely activated and had irregular cell bodies in one third of the TK group patients (Figure 2p). This feature was also found in about half of the DE group (Figure $2 q$ ), whereas the keratocytes of the normal group were predominantly inactive and had oval-shaped nuclei (Figure 2r).

Moreover, in vivo LSCM showed other morphological characteristics of the TK subjects, which are summarized in Figure 3a. Highly reflective cell borders in the superficial epithelium were observed in 9 of the 16 patients (56.3\%) in the TK group and in 5 of the 16 patients $(31.3 \%)$ in the DE group $(P>0.05$, Figure $3 b)$. Epithelial cells with highly reflective nuclei in the basal cell layer were noted a lot in patients in both the TK and DE groups $(62.5 \%$ in TK vs $31.3 \%$ in DE, $P>0.05$, Figure 3c). Most notably, the epithelium had a snow-like appearance in 12 of the 16 TK patients $(75.0 \%)$, but none of the DE patients $(P<0.05$, Figure $3 \mathrm{~d})$. In addition, activated keratocytes were observed in 6 of the 16 TK patients (37.5\%) and in 9 of the $16 \mathrm{DE}$ patients $(56.3 \%)(P>0.05$, Figure $3 e)$.

\section{Discussion}

The current study made comparison on the grades of area, density, and distribution of corneal fluorescein punctate staining in subjects with SPK. The results revealed that the grade of the area was higher in subjects with TK than those diagnosed as DE, which suggests that the ocular surface destruction was more widespread in TK. Intriguingly, we also found that the fluorescein staining mainly involved the middle and lower thirds of the cornea in both the TK and DE groups, whereas the upper part of the cornea rarely suffered and was only involved in extensively impaired corneas. This result is in accordance with a previous study about TK, which reported that the greatest staining usually occurred inferiorly or inferonasally. ${ }^{3}$ This finding is probably due to gravitational effects tear drainage and the ease with which drops placed in the inferior cornea.

It is notable that the majority of the eyes with TK had a snow-like appearance in the corneal epithelium, which was not found in the DE patients. Scattered hyperreflective round dots with obscure outlines and of various sizes had a snow-like pattern and accumulated into large aggregates in some areas of the corneal epithelium. The deposits had no distinct cellular features that resembled inflammatory cells or Langerhans cells. This characteristic might be unique to TK because a similar confocal appearance has not been described in human corneal epithelium. However, in corneal stroma, BoÈhnkeet al ${ }^{11}$ reported that numerous 'micro-dots' were present after a long period of soft contact lens wear, and Rodrigues et al ${ }^{12}$ found that 'whitish crystalline deposits' appeared posterior to a 5-mm polymethyl methacrylate intracorneal lens. According to their analyses, the nature of these structures had not been determined, but it was possible that the deposits consisted of lipofuscein granules that were produced by keratocytes as a nonspecific response to stress. A portion of these granules might be deposited in the extracellular compartment, where they stay and accumulate over time. As for the epithelium deposits found in our study, we propose the following two hypotheses: (1) a number of keratocytes in the stroma were activated, as suggested by the visualization of keratocyte cell bodies and processes, and yielded lipofuscein granules, which penetrated through the porous Bowman's membrane and lipophilic epithelial basement membrane and deposited in the basal epithelial layer; (2) because BAC, the most widely used preservative, has been proven to be capable of damaging the corneal epithelium by affecting cell-to-cell junctions, cell shapes, and microvillus, which eventually leads to cell necrosis ${ }^{13-15}$ the debris deposits possibly resulted from cell lyses.

Apart from snow-like hyper-reflective deposits, the morphological changes of TK include extensive hyperreflective cellular outlines of the superficial epithelial layer and highly reflective nuclei within basal epithelial cells. The bright cell borders of the epithelial cells possibly indicate a loss of contact and the process of desquamation. ${ }^{16}$ These two changes might demonstrate that a large amount of epithelial cells in the superficial layer tended to drop off the eye surface, which stimulated the proliferation of basal epithelial cells to compensate for the reduction in epithelial cells. In addition, poor attachment of the superficial epithelium can also lead to epithelial erosion, which exposes the underlying, smaller wing cells; this is possibly observed as punctate fluorescein staining with slit-lamp biomicroscopy. ${ }^{13,14,17}$ Significantly decreased density of superficial epithelial cells is another characteristic of TK. A previous study showed that the density of superficial epithelial cells was reduced in all glaucomatous patients, except in patients who used preservative-free eye drops. ${ }^{18}$ Therefore, we deduce that the reduction of cellular density may also be attributed to the damaging effects of eye drops on the external epithelium because BAC can disrupt epithelial tight junctions and induce cell apoptosis in a dosedependent manner. ${ }^{19}$

Previous studies have shown that topical medication can reduce the number of sub-basal nerves in the cornea while increasing their tortuosity. ${ }^{18}$ However, in the current study, we found that the corneas of patients with TK had less abundant and less tortuous sub-basal nerves compared with either the DE or the normal groups. Previous studies on the denervation effect of BAC on corneas demonstrated that the number of sub-basal nerves was reduced and that based on esthesiometry, the 
corneal sensitivity decreased in the groups that used preservative-containing drugs versus the group that used preservative-free drugs and the control group. ${ }^{18}$ These findings indicate the loss of sub-basal nerve density may contribute to the development of ocular surface disorders because corneal sensory innervations have an important

a

\begin{tabular}{llll}
\hline Presentation & TK & DE & P Value \\
\hline Highly reflective cell border in the $9 / 16(56.3 \%)$ & $5 / 16(31.3 \%)$ & 0.285 \\
superficial epithelium & & & \\
Highly reflective nucleus in the basal & $10 / 16(62.5 \%)$ & $5 / 16(31.3 \%)$ & 0.156 \\
epithelium & & & \\
Snow-like appearance of epithelium & $12 / 16(75.0 \%)$ & $0 / 16(0 \%)$ & $0.000^{*}$ \\
Activation of keratocytes & $6 / 16(37.5 \%)$ & $9 / 16(56.3 \%)$ & 0.479 \\
\hline
\end{tabular}

† P values derived from Exact Fisher Test

${ }^{*} \mathrm{P}<0.01$

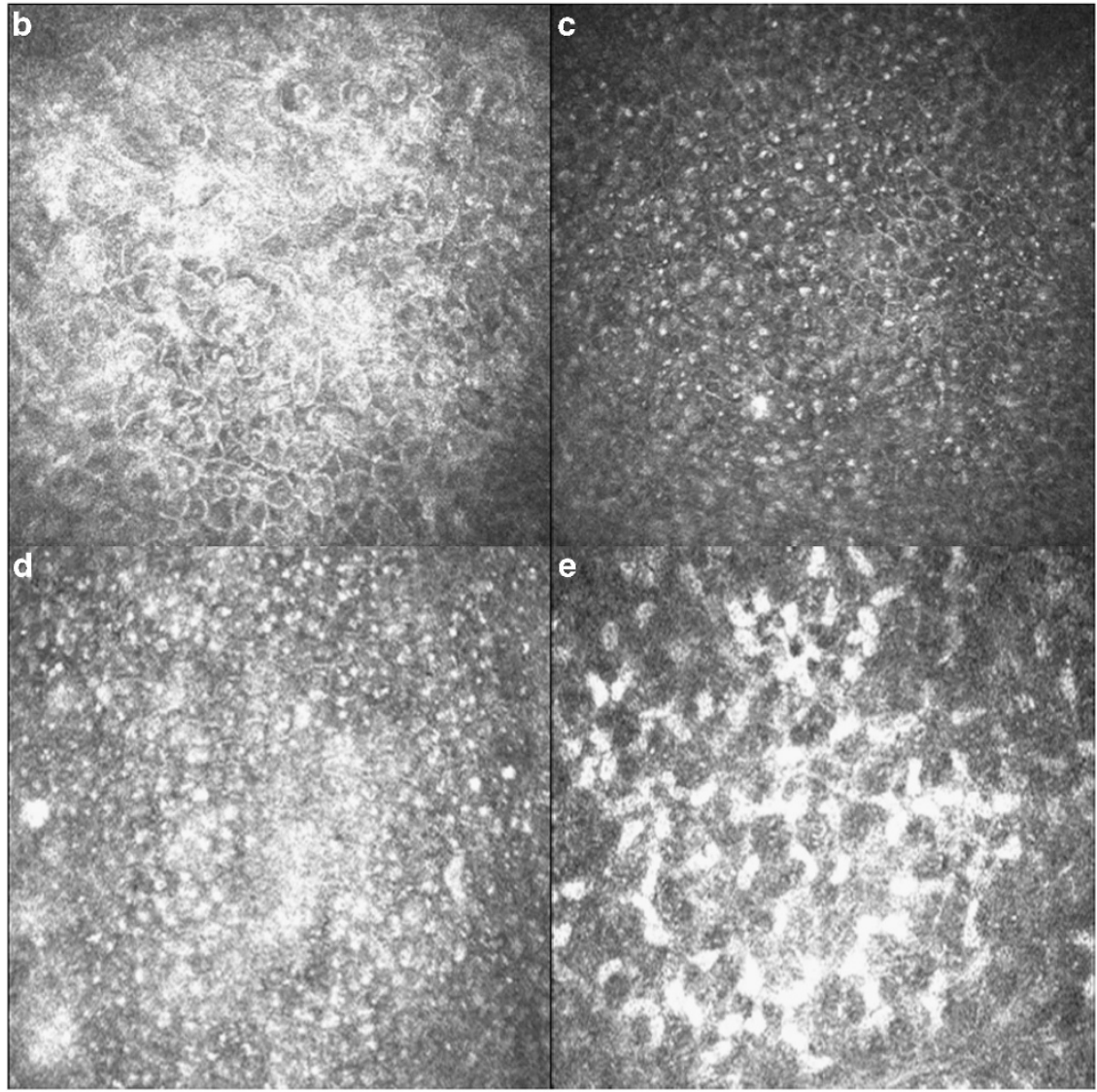

Figure 3 Characteristic presentations in in vivo LSCM Images of TK. Representative in vivo confocal microscopic images obtained from TK patients were summarized (a). Most of the TK cases showed highly reflective cell borders in the superficial epithelium (b), highly reflective nuclei in the basal epithelium (c), a snow-like pattern in the epithelium (d), and activated keratocytes (e). 
influence on corneal trophism and contribute to the maintenance of corneal integrity. ${ }^{20,21}$

With the help of in vivo confocal microscopy, TK can be distinguished from other conditions. In herpes simplex virus infection cases, reduced nerve density or even no visible sub-basal plexus can be seen, whereas the epithelial cell density has no significant changes. ${ }^{22}$ Increased density of dendritic-like cells has been reported in patients with bacterial keratitis. ${ }^{23}$ Acanthamoeba keratitis is characterized by the cysts with a double-wall appearance. ${ }^{24}$ In the anterior stroma of the scarring cornea, there were hyperrective deposits, forming wide bands, ${ }^{25}$ which is easily differentiated from the diffuse snow-like pattern of deposits in the epithelium in TK. In vivo confocal microscopy in corneas affected by recurrent corneal erosion (RCE) mostly shows strangeshaper or diminished sub-basal nerves, and in a few cases, highly reflective basal epithelial cells with intracellular deposits (not scattered hyper-reflective dots of various sizes) in the basal epithelium and sub-basal microfolds and streaks. ${ }^{26}$

Some limitations of this study should be addressed. First, the in vivo confocal microscopy measurements were taken at the fluorescein staining zone that was either in the center or the periphery of the cornea, despite the fact the two parts are inherently different in confocal functioning. However, this difference maybe minor relative to the differences between different diseases. Second, we had a small sample size of the TK group due to the increasing standardization of clinical application of eye drops in China in recent years; a larger sample size would allow for more definitive conclusions to be made.

In conclusion, SPK in TK patients was characterized with more widespread punctate staining, a lower density of superficial epithelial cells and sub-basal nerves when compared with DE, and a typical snow-like pattern of deposits in the epithelium by LSCM. LSCM might be a useful tool in differentiating the etiology of SPK, as well as the early diagnosis of TK.

\section{Summary}

What was known before

- Toxic keratopathy (TK) involves corneal damage or dysfunction that results from exposure to substances that are disruptive to corneal anatomy or metabolism. TK can be induced by inappropriate use of all kinds of eye drops. However, there has not been any effective way to diagnose it.

What this study adds

- SPK in TK patients was characterized with more widespread punctate stainings, a lower density of superficial epithelial cells and sub-basal nerves, when compared with DE, and a typical snow-like pattern of deposits in the epithelium by LSCM.

\section{Conflict of interest}

The authors declare no conflict of interest.

\section{Acknowledgements}

This work was supported by grants from National Science and Technology Research Program, the Ministry of Science, and Technology, China (2012BAI08B01). The sponsor or funding organization had no role in the design or conduct of this research. The authors declare no financial relationship with the organization that sponsored the research.

\section{References}

1 DeRosa AJ. Toxic keratopathy. Int Ophthalmol Clin 1998; 38(4): 15-22.

2 Fraunfelder FW. Corneal toxicity from topical ocular and systemic medications. Cornea 2006; 25(10): 1133-1138.

3 Schwab IR, Abbott RL. Toxic ulcerative keratopathy. An unrecognized problem. Ophthalmology 1989; 96(8): 1187-1193.

4 Coster D. Duane's Clinical Ophthalmology. Lippincott: Philadelphia, 1991.

5 Kobayashi A, Yokogawa H, Sugiyama K. In vivo laser confocal microscopy findings of Thygeson superficial punctate keratitis. Cornea 2011; 30(6): 675-680.

6 Miyata K, Amano S, Sawa M, Nishida T. A novel grading method for superficial punctate keratopathy magnitude and its correlation with corneal epithelial permeability. Arch Ophthalmol 2003; 121(11): 1537-1539.

7 Uchino Y, Uchino M, Dogru M, Ward S, Yokoi N, Tsubota K. Changes in dry eye diagnostic status following implementation of revised Japanese dry eye diagnostic criteria. Jpn J Ophthalmol 2012; 56(1): 8-13.

8 [No authors listed]. Methodologies to diagnose and monitor dry eye disease: report of the Diagnostic Methodology Subcommittee of the International Dry Eye WorkShop (2007). Ocul Surf 2007; 5(2): 108-152.

9 Oliveira-Soto L, Efron N. Morphology of corneal nerves using confocal microscopy. Cornea 2001; 20(4): 374-384.

10 Vanathi M, Tandon R, Sharma N, Titiyal JS, Pandey RM, Vajpayee RB. In vivo slit scanning confocal microscopy of normal corneas in Indian eyes. Indian J Ophthalmol 2003; 51(3): 225-230.

11 Bohnke M, Masters BR. Long-term contact lens wear induces a corneal degeneration with microdot deposits in the corneal stroma. Ophthalmology 1997; 104(11): 1887-1896.

12 Rodrigues MM, McCarey BE, Waring GR, Hidayat AA, Kruth HS. Lipid deposits posterior to impermeable intracorneal lenses in rhesus monkeys: clinical, histochemical, and ultrastructural studies. Refract Corneal Surg 1990; 6(1): 32-37.

13 Ghosh M, McCulloch C. Whorl-like degeneration of corneal epithelium a clinico-pathologic study. Can J Ophthalmol 1978; 13(2): 110-119.

14 Risco JM, Millar LC. Ultrastructural alterations in the endothelium in a patient with topical anesthetic abuse keratopathy. Ophthalmology 1992; 99(4): 628-633. 
15 Hosal BM, Ornek N, Zilelioglu G, Elhan AH. Morphology of corneal nerves and corneal sensation in dry eye: a preliminary study. Eye (Lond) 2005; 19(12): 1276-1279.

16 Bohnke M, Masters BR. Confocal microscopy of the cornea. Prog Retin Eye Res 1999; 18(5): 553-628.

17 Pauly A, Brignole-Baudouin F, Labbe A, Liang H, Warnet JM, Baudouin C. New tools for the evaluation of toxic ocular surface changes in the rat. Invest Ophthalmol Vis Sci 2007; 48(12): 5473-5483.

18 Martone G, Frezzotti P, Tosi GM, Traversi C, Mittica V, Malandrini A et al. An in vivo confocal microscopy analysis of effects of topical antiglaucoma therapy with preservative on corneal innervation and morphology. Am J Ophthalmol 2009; 147(4): 725-735.

19 Pauly A, Meloni M, Brignole-Baudouin F, Warnet JM, Baudouin C. Multiple endpoint analysis of the 3Dreconstituted corneal epithelium after treatment with benzalkonium chloride: early detection of toxic damage. Invest Ophthalmol Vis Sci 2009; 50(4): 1644-1652.

20 Nishida T, Chikama T, Sawa M, Miyata K, Matsui T, Shigeta K. Differential contributions of impaired corneal sensitivity and reduced tear secretion to corneal epithelial disorders. Jpn J Ophthalmol 2012; 56(1): 20-25.

21 Benitez DCJ, Wasfy MA, Fernandez C, Garcia-Sanchez J. An in vivo confocal masked study on corneal epithelium and subbasal nerves in patients with dry eye. Invest Ophthalmol Vis Sci 2004; 45(9): 3030-3035.

22 Rosenberg ME, Tervo TM, Muller LJ, Moilanen JA, Vesaluoma MH. In vivo confocal microscopy after herpes keratitis. Cornea 2002; 21(3): 265-269.

23 Su PY, Hu FR, Chen YM, Han JH, Chen WL. Dendritiform cells found in central cornea by in vivo confocal microscopy in a patient with mixed bacterial keratitis. Ocul Immunol Inflamm 2006; 14(4): 241-244.

24 Bourcier T, Dupas B, Borderie V, Chaumeil C, Larricart P, Baudouin $\mathrm{C}$ et al. Heidelberg retina tomograph II findings of Acanthamoeba keratitis. Ocul Immunol Inflamm 2005; 13(6): 487-492.

25 Song $\mathrm{P}$, Wang S, Zhang $\mathrm{P}$, Sui W, Zhang Y, Liu T et al. The superficial stromal scar formation mechanism in keratoconus: a study using laser scanning in vivo confocal microscopy. Biomed Res Int 2016; 2016: 7092938.

26 Rosenberg ME, Tervo TM, Petroll WM, Vesaluoma MH. In vivo confocal microscopy of patients with corneal recurrent erosion syndrome or epithelial basement membrane dystrophy. Ophthalmology 2000; 107(3): 565-573.

(c) (i) $\odot$ This work is licensed under a Creative Commons Attribution-NonCommercialNoDerivs 4.0 International License. The images or other third party material in this article are included in the article's Creative Commons license, unless indicated otherwise in the credit line; if the material is not included under the Creative Commons license, users will need to obtain permission from the license holder to reproduce the material. To view a copy of this license, visit http://creativecommons.org/licenses/by-nc-nd/4.0/

(C) The Author(s) 2017 